\title{
Self-bound droplet of Bose and Fermi atoms in one dimension: Collective properties in mean-field and Tonks-Girardeau regimes
}

\author{
Luca Salasnich, ${ }^{1, *}$ Sadhan K. Adhikari, ${ }^{2, \dagger}$ and Flavio Toigo ${ }^{3, \dagger}$ \\ ${ }^{1}$ CNISM and CNR-INFM, Unità di Padova, Via Marzolo 8, 35131 Padova, Italy \\ ${ }^{2}$ Instituto de Física Teórica, UNESP-São Paulo State University, 01.405-900 São Paulo, São Paulo, Brazil \\ ${ }^{3}$ Dipartimento di Fisica "G. Galilei” and CNISM, Università di Padova, Via Marzolo 8, 35131 Padova, Italy
}

(Received 10 January 2007; published 20 February 2007)

\begin{abstract}
We investigate a dilute mixture of bosons and spin-polarized fermions in one dimension. With an attractive Bose-Fermi scattering length the ground state is a self-bound droplet, i.e., a Bose-Fermi bright soliton where the Bose and Fermi clouds are superimposed. We find that the quantum fluctuations stabilize the Bose-Fermi soliton such that the one-dimensional bright soliton exists for any finite attractive Bose-Fermi scattering length. We study density profile and collective excitations of the atomic bright soliton showing that they depend on the bosonic regime involved: mean-field or Tonks-Girardeau.
\end{abstract}

DOI: 10.1103/PhysRevA.75.023616

PACS number(s): 03.75.Ss, 03.75.Hh, 64.75.+g

\section{INTRODUCTION}

Ultracold vapors of alkali-metal atoms, such as ${ }^{87} \mathrm{Rb}$, ${ }^{85} \mathrm{Rb},{ }^{40} \mathrm{~K},{ }^{23} \mathrm{Na},{ }^{6} \mathrm{Li},{ }^{7} \mathrm{Li}$, etc. are now actively studied in the regime of deep Bose and Fermi degeneracy [1-5]. Trapped Bose-Fermi mixtures, with Fermi atoms in a single hyperfine state, have been investigated by various authors both theoretically [6-12] and experimentally [13-18]. Recently, it has been predicted that self-bound droplets, also called atomic bright solitons, can be formed within a mixture of degenerate Bose-Fermi gases provided the gases attract each other strongly enough and that there is an external transverse confinement [19-21]. Formation of bright solitons in a dilute spin-polarized Fermi gas is prevented by Pauli repulsion. The formation of bright soliton in a Bose-Fermi mixture is related to the fact that the system can lower its energy by forming high-density regions (bright solitons) when the Bose-Fermi attraction is sufficient to overcome the Pauli repulsion among Fermi atoms and any possible repulsion among the Bose atoms. A common point of these papers [19-21] is that the Fermi cloud is three-dimensional (3D). In fact, for not too strong Bose-Bose repulsion the transverse width of the Fermi component significantly exceeds the transverse width of the Bose component [19-21].

In the strict one-dimensional (1D) regime, the Bose-Fermi mixture requires an appropriate theoretical description. The exponent of the power-law, which describes the bulk energy of a Fermi gas as a function of its density depends on the dimensionality (see, for instance, Ref. [22]). In addition, even at zero temperature, the 1D Bose gas can never be a true Bose-Einstein condensate due to phase fluctuations $[1,23]$. For a repulsive 1D Bose gas, one must distinguish two regimes: a quasi-Bose-Einstein condensate (BEC) regime, well described by the 1D Gross-Pitaevskii equation

*URL: http://www.padova.infm.it/salasnich. Electronic address: salasnich@pd.infn.it

${ }^{\dagger}$ URL: http://www.ift.unesp.br/users/adhikari. Electronic address: adhikari@ift.unesp.br

${ }^{\ddagger}$ Electronic address: toigo@pd.infn.it with positive nonlinearity [23], and a Tonks-Girardeau (TG) regime at very low densities, where the 1D bosons behave as 1D ideal fermions [24,25]. An attractive 1D Bose gas is instead well described by the Hartree mean-field theory $[26,27]$, i.e., the 1D Gross-Pitaevskii equation with negative nonlinearity [28].

The existence of the TG regime above has recently been experimentally confirmed [29] in a study of the 1D degenerate ${ }^{87} \mathrm{Rb}$ system. In a subsequent study of this system [30], the 1D Bose gas in the TG regime has been found to possess the peculiar property of not attaining a thermal equilibrium even after thousands of collisions. This is often termed fermionization of $1 \mathrm{D}$ bosons in the TG regime. It is well known that due to Pauli principle the spin-polarized trapped fermions do not interact at low temperature and hence fail to reach a thermal equilibrium necessary for evaporative cooling leading to a degenerate state. The necessary thermal equilibrium was attained only in Bose-Fermi [16-18] or FermiFermi [31] mixtures through collision between bosons and fermions or between fermions in different quantum states, respectively.

In this paper we consider a Bose-Fermi mixture strongly confined by a $2 \mathrm{D}$ harmonic potential in the transverse cylindric radial coordinate. The ensuing effective $1 \mathrm{D}$ system is described in the quantum hydrodynamical approximation, i.e., the time-dependent density-functional approach based on real hydrodynamic variables or complex scalar fields. Quantum hydrodynamics is very useful for the study of static and collective properties of a Bose-Fermi mixture and it has been used successfully in 3D [20,21,32] for a description of bright and dark solitons and collapse. We investigate the 1D mixture of bosons and spin-polarized fermions by using an effective 1D Lagrangian [1,33-35]. A Gaussian variational approach is adopted to derive axial static and dynamical properties of the mixture with attractive Bose-Fermi scattering length $\left(a_{b f}<0\right)$. The solution of the variational scheme was found to be in satisfactory agreement with the accurate numerical solution of the hydrodynamic equations. We find that a self-bound droplet, i.e., a Bose-Fermi bright soliton, exists also for very small values of $\left|a_{b f}\right|$. In this case the axial width of the Fermi component is very large while the axial 
width of the Bose component depends on the sign and magnitude of the Bose-Bose scattering length $a_{b}$. Remarkably, the TG regime is essential to preserve a localized BoseFermi soliton for very small $\left|a_{b f}\right|$; in fact, for a repulsive Bose-Bose interaction in the quasi-BEC 1D regime the theory predicts a minimum value of $\left|a_{b f}\right|$ below which the mixture is uniform, i.e., fully delocalized. For large values of $\left|a_{b f}\right|$ the Bose-Fermi system is self-confined in a very narrow region and therefore the local axial densities of bosons and fermions strongly increase. We must remember, however, that above a critical axial density the Fermi system is no more strictly one dimensional, and the same happens for repulsive bosons.

The paper is organized as follows. In Sec. II we present the model used to study the degenerate Bose-Fermi system. Then we derive a set of coupled equations for the mixture starting from a Lagrangian density. In Sec. III, by using a Gaussian variational ansatz, we demonstrate that for an attractive Bose-Fermi interaction, the ground state of the model is a self-bound bright soliton (in the absence of an external longitudinal trap). In Sec. IV we present a study of the system in the quasi-BEC regime and in the TG regime for attractive Bose-Fermi interaction. These results are further explored in Sec. V considering a single Fermi atom inside the Bose cloud. In Sec. VI we consider the problem of coupled breathing oscillations of the Bose-Fermi system and calculate the frequencies of these oscillations. Finally, in Sec. VII we present a brief summary of our investigation and discuss the experimental conditions necessary to achieve the 1D Bose-Fermi soliton.

\section{BOSE-FERMI LAGRANGIAN FOR ONE-DIMENSIONAL HYDRODYNAMICS}

We consider a mixture of $N_{b}$ bosons of mass $m_{b}$ and $N_{f}$ spin-polarized fermions of mass $m_{f}$ at zero temperature trapped by a tight cylindrically symmetric harmonic potential of frequency $\omega_{\perp}$ in the transverse direction. We assume factorization of the transverse degrees of freedom. It is justified in 1D confinement where, regardless of the longitudinal behavior or statistics, the transverse spatial profile is that of the single-particle ground state [10,35-38]. The transverse width of the atom distribution is given by the characteristic harmonic length of the single-particle ground state: $a_{\perp j}$ $=\sqrt{\hbar /\left(2 m_{j} \omega_{\perp}\right)}$, with $j=b, f$. The atoms have an effective 1D behavior at zero temperature if their chemical potentials are much smaller than the transverse energy $\hbar \omega_{\perp}[10,35]$.

We use a hydrodynamic effective Lagrangian to study the static and collective properties of the 1D Bose-Fermi mixture. In the rest of the paper all quantities are dimensionless. In particular, lengths are in units of $a_{\perp b}$, linear densities in units of $a_{\perp b}^{-1}$, times in units of $\omega_{\perp}^{-1}$, and energies in units of $\hbar \omega_{\perp}$. The Lagrangian density $\mathcal{L}$ of the mixture reads

$$
\mathcal{L}=\mathcal{L}_{b}+\mathcal{L}_{f}+\mathcal{L}_{b f}
$$

The term $\mathcal{L}_{b}$ is the bosonic Lagrangian, defined as

$$
\mathcal{L}_{b}=\psi_{b}^{*}\left(i \partial_{t}+\partial_{z}^{2}\right) \psi_{b}-\left|\psi_{b}\right|^{6} G\left(\frac{g_{b}}{2\left|\psi_{b}\right|^{2}}\right)-V_{b}\left|\psi_{b}\right|^{2},
$$

where $\psi_{b}(z, t)$ is the hydrodynamic field of the Bose gas, such that $n_{b}(z, t)=\left|\psi_{b}(z, t)\right|^{2}$ is the $1 \mathrm{D}$ density and $v_{b}(z, t)$ $=i \partial_{z} \ln \left[\psi_{b}(z, t) /\left|\psi_{b}(z, t)\right|\right]$ is its velocity. Here $g_{b}=2 a_{b} / a_{\perp b}$ is the scaled interatomic strength with $a_{b}$ the Bose-Bose scattering length. We take $\left|g_{b}\right|<1$ to avoid the confinementinduced resonance [39]. Interacting bosons are onedimensional if $g_{b} n_{b} \ll 1$ [35-37]. For $x>0$ the function $G(x)$ is the so-called Lieb-Liniger function, defined as the solution of a Fredholm equation and such that $G(x) \simeq x$ for $0<x \ll 1$ and $G(x) \simeq \pi^{2} / 3$ for $x \gg 1$ [24]. For $x<0$ we set $G(x)=x$ [28,35]. $V_{b}(z)$ is the longitudinal external potential acting on the bosons. In the static case the Lagrangian density $\mathcal{L}_{b}$ reduces exactly to the energy functional recently introduced by Lieb, Seiringer, and Yngvason [40]. In addition, $\mathcal{L}_{b}$ has been successfully used to determine the collective oscillation of the 1D Bose gas with longitudinal harmonic confinement [35].

The fermionic Lagrangian density $\mathcal{L}_{f}$ is given instead by

$$
\mathcal{L}_{f}=\psi_{f}^{*}\left(\partial_{t}+\lambda_{m} \partial_{z}^{2}\right) \psi_{f}-\frac{\pi^{2} \lambda_{m}}{3}\left|\psi_{f}\right|^{6}-V_{f}\left|\psi_{f}\right|^{2},
$$

where $\lambda_{m}=m_{b} / m_{f}$ and $\psi_{f}(z, t)$ is the hydrodynamic field of the 1D spin-polarized Fermi gas, such that $n_{f}(z, t)$ $=\left|\psi_{f}(z, t)\right|^{2}$ is the 1D fermionic density and $v_{f}(z, t)$ $=i \lambda_{m} \partial_{z} \ln \left[\psi_{f}(z, t) /\left|\psi_{f}(z, t)\right|\right]$ is the velocity of the Fermi gas. The noninteracting fermions are $1 \mathrm{D}$ if $\left(\pi^{2} \lambda_{m} / 2\right) n_{f}^{2} \ll 1[10]$. $V_{f}(z)$ is the longitudinal external potential acting on fermions. In the static case and with $V_{f}(z)=0$ the Lagrangian $\mathcal{L}_{f}$ gives the correct energy density of a uniform and noninteracting 1D Fermi gas. More generally, the Euler-Lagrange equation of $\mathcal{L}_{f}$ yields the hydrodynamic equations of the $1 \mathrm{D}$ Fermi gas [34].

Finally, the Lagrangian density $\mathcal{L}_{b f}$ of the Bose-Fermi interaction reads

$$
\mathcal{L}_{b f}=-g_{b f}\left|\psi_{b}\right|^{2}\left|\psi_{f}\right|^{2},
$$

where $g_{b f}=2 a_{b f} / a_{\perp b}$ is the scaled interatomic strength between bosons and fermions, with $a_{b f}$ the Bose-Fermi scattering length [10].

Euler-Lagrange equations of the Lagrangian $\mathcal{L}$ provide the two coupled partial differential equations for $\psi_{b}$ and $\psi_{f}$,

$$
\begin{aligned}
i \partial_{t} \psi_{b}= & {\left[-\partial_{z}^{2}+3\left|\psi_{b}\right|^{4} G\left(\frac{g_{b}}{2\left|\psi_{b}\right|^{2}}\right)\right.} \\
& \left.-\frac{1}{2} g_{b}\left|\psi_{b}\right|^{2} G^{\prime}\left(\frac{g_{b}}{2\left|\psi_{b}\right|^{2}}\right)+V_{b}+g_{b f}\left|\psi_{f}\right|^{2}\right] \psi_{b}, \\
i \partial_{t} \psi_{f}= & {\left[-\lambda_{m} \partial_{z}^{2}+\pi^{2} \lambda_{m}\left|\psi_{f}\right|^{4}+V_{f}+g_{b f}\left|\psi_{b}\right|^{2}\right] \psi_{f} . }
\end{aligned}
$$

For $g_{b f}=0$ and $0<g_{b}<1$, the first partial differential equation (5) reduces, in the regime $g_{b} / n_{b} \ll 1$, to the familiar mean-field 1D Gross-Pitaevskii equation [1], i.e., to the 1D cubic nonlinear Schrödinger equation describing a quasiBEC. Instead, in the regime where everywhere $g_{b} / n_{b} \gg 1$, Eq. (5) for bosons becomes the quintic nonlinear 
Schrödinger equation proposed by Kolomeisky et al. [33] for the dynamics of a TG gas, which is formally equivalent to Eq. (6) describing the 1D noninteracting Fermi gas. Actually, Girardeau and Wright [41] have shown that this quintic nonlinear Schrödinger equation overestimates the coherence in interference patterns at a small number of particles. Nevertheless, Minguzzi et al. [34] have found that this quintic equation is quite accurate in describing the density profile and the collective oscillations of the 1D ideal Fermi gas with longitudinal harmonic confinement. If we define $G(x)=x$ for $x<0$ then, when $g_{b f}=0$ and $g_{b}<0$ Eq. (5) reduces to the mean-field 1D Gross-Pitaevskii equation with attractive (negative) nonlinearity, which describes quite accurately the attractive 1D Bose gas $[27,28]$.

\section{SELF-BOUND SOLUTION: BOSE-FERMI BRIGHT SOLITON}

In the remaining part of the paper we set $V_{b}(z)=V_{f}(z)$ $=0$ and investigate the case of a negative Bose-Fermi scattering length $\left(g_{b f}<0\right)$. We use a time-dependent variational ansatz for the fields $\psi_{j}(z, t)$ to determine the conditions under which a self-bound droplet of 1D bosons and fermions exists. In particular, we investigate the two main regimes of 1D bosons: the quasi-BEC regime and the TG regime. For the two fields $\psi_{j}(z, t)$, with $j=b, f$, we use the following Gaussian ansatz:

$$
\psi_{j}=\frac{N_{j}^{1 / 2}}{\pi^{1 / 4} \sigma_{j}^{1 / 2}} \exp \left(-\frac{\left(z-z_{j}\right)^{2}}{2 \sigma_{j}^{2}}+i \phi_{j} z+i \theta_{j} z^{2}\right),
$$

where the time-dependent variational parameters are the longitudinal widths $\sigma_{j}(t)$, the centers of mass $z_{j}(t)$, and the slopes $\phi_{j}(t)$ and curvatures $\theta_{j}(t)$ of the phase. It is obvious that the tails of the Gaussian $n_{b}(z, t)=\left|\psi_{b}(z, t)\right|^{2}$ given by Eq. (7) are locally in the TG regime but, in our terminology, a nonuniform cloud of bosons is in the TG regime only if everywhere its local density $n_{b}(z, t)$ satisfies the condition $g_{b} / n_{b}(z, t) \gg 1$.

We insert the Gaussian fields $\psi_{j}(z, t)$ into the Lagrangian $\mathcal{L}$ and integrate over the spatial variable $z$ and get an effective Lagrangian $[42,43]$, which depends on $\sigma_{j}(t), z_{j}(t), \phi_{j}(t)$, $\theta_{j}(t)$ and their time derivatives. By writing the eight EulerLagrange equations one finds that the slopes $\phi_{j}(t)$ and the curvatures $\theta_{j}(t)$ of the fields $\psi_{j}(z, t)$ can be obtained from the widths $\sigma_{j}(t)$ and the center coordinates $z_{j}(t)$ through the equations

$$
\phi_{j}=-\dot{z}_{j}-2 \theta_{j} z_{j}, \quad \theta_{j}=-\frac{\dot{\sigma}_{j}}{2 \sigma_{j}},
$$

with $j=b, f$. The equations of motion of the parameters $\sigma_{j}(t)$ and $z_{j}(t)$ do not depend on the phase parameters $\phi_{j}(t)$ and $\theta_{j}(t)[42,43]$. They are the "classical" equations of motion of a system with effective Lagrangian

$$
L=T-E,
$$

where

$$
T=\frac{N_{b}}{2}\left(\dot{\sigma}_{b}^{2}+2 \dot{z}_{b}^{2}\right)+\frac{N_{f} \lambda_{m}}{2}\left(\dot{\sigma}_{f}^{2}+2 \dot{z}_{f}^{2}\right)
$$

is the effective kinetic energy and

$$
E=E_{b}+E_{f}+E_{b f}
$$

is the effective potential energy of the system. The term $E_{b}$ involves a complicated integral of the Lieb-Liniger function $G(x)$, namely,

$$
E_{b}=\frac{N_{b}}{2 \sigma_{b}^{2}}+\frac{N_{b}^{3}}{\pi^{3 / 2} \sigma_{b}^{2}} \int_{-\infty}^{+\infty} e^{-3 y^{2}} G\left(\frac{g_{b} \sigma_{b}}{2 N_{b}} e^{y^{2}}\right) d y .
$$

The other two terms, $E_{f}$ and $E_{b f}$, are given by

$$
E_{f}=\frac{N_{f} \lambda_{m}}{2 \sigma_{f}^{2}}+\frac{N_{f}^{3} \pi \lambda_{m}}{3 \sqrt{3} \sigma_{f}^{2}},
$$

and

$$
E_{b f}=\frac{g_{b f} N_{b} N_{f}}{\sqrt{\pi} \sigma_{b f}} \exp \left[-\frac{\left(z_{b}-z_{f}\right)^{2}}{\sqrt{\sigma_{b}^{2}+\sigma_{f}^{2}}}\right] .
$$

We stress that the potential energy (11) of the effective Lagrangian (9) can be easily obtained from ansatz (7) without including the phase parameters $\phi_{j}(t)$ and $\theta_{j}(t)$. On the contrary, to get the kinetic energy term (10) it is necessary to include in the ansatz the four phase parameters of Eq. (7) $[42,43]$. The kinetic term is essential to calculate the dynamical properties of the mixture, such as the collective oscillations considered in Sec. VI.

The stable stationary state of the system is found by minimizing the effective potential energy

$$
\begin{aligned}
& \frac{\partial E}{\partial z_{j}}=0, \quad j=b, f, \\
& \frac{\partial E}{\partial \sigma_{j}}=0, \quad j=b, f .
\end{aligned}
$$

Equations (15) lead to $z_{b}=z_{f}$ and without loss of generality we set $z_{b}=z_{f}=0$. Equations (16) can then be rewritten as

$$
\begin{gathered}
1+\frac{2 N_{b}^{2}}{\pi^{3 / 2}} \int_{-\infty}^{+\infty} e^{-3 y^{2}} G\left(\frac{g_{b} \sigma_{b}}{2 N_{b}} e^{y^{2}}\right) d y \\
-\frac{g_{b} N_{b} \sigma_{b}}{2 \pi^{3 / 2}} \int_{-\infty}^{+\infty} e^{-2 y^{2}} G^{\prime}\left(\frac{g_{b} \sigma_{b}}{2 N_{b}} e^{y^{2}}\right)=\frac{-g_{b f} N_{f} \sigma_{b}^{4}}{\sqrt{\pi} \sigma_{b f}^{3}} \\
\lambda_{m}+\frac{2 N_{f}^{2} \pi \lambda_{m}}{3 \sqrt{3}}=\frac{-g_{b f} N_{b} \sigma_{f}^{4}}{\sqrt{\pi} \sigma_{b f}^{3}}
\end{gathered}
$$

where $\sigma_{b f}=\sqrt{\sigma_{b}^{2}+\sigma_{f}^{2}}$. From Eqs. (17) and (18) one can determine the widths $\sigma_{b}$ and $\sigma_{f}$ at equilibrium. Stability requires that the Hessian matrix of the second partial derivatives of the effective potential energy $E\left(\sigma_{j}\right)$ is positive definite; equivalently, the Gaussian curvature 


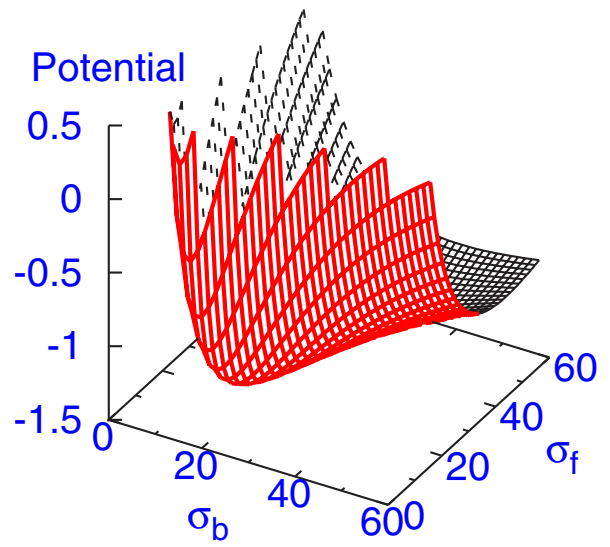

FIG. 1. (Color online) Effective potential energy $E$ of Eq. (11) as a function of soliton widths $\sigma_{b}$ and $\sigma_{f}$ for parameters: $\lambda_{m}=1$, $N_{b}=100, N_{f}=10, g_{b}=0.01$, and $g_{b f}=-0.2$. The potential has a minimum at $\sigma_{b}=16.90$ and $\sigma_{f}=21.84$. Lengths are in units of $a_{\perp b}$ $=\sqrt{\hbar /\left(2 m_{b} \omega_{\perp}\right)}$ and energy is in units of $\hbar \omega_{\perp}$.

$$
K_{G}=\frac{\partial^{2} E}{\partial \sigma_{b}^{2}} \frac{\partial^{2} E}{\partial \sigma_{f}^{2}}-\left(\frac{\partial^{2} E}{\partial \sigma_{b} \partial \sigma_{f}}\right)^{2}
$$

must be positive. An inspection of Eqs. (17) and (18) shows that there are stable solutions only for $g_{b f} \leqslant 0$. For $g_{b f}=0$ and $g_{b}>0$, the solutions are $\sigma_{b}=\sigma_{f}=+\infty$, corresponding to infinitely extended, uniform bosonic and fermionic clouds, while for $g_{b f}=0$ and $g_{b}<0$, the fermionic 1D density is uniform while the bosonic cloud is localized with $\sigma_{b}$ $=2 \sqrt{2 \pi} /\left(\left|g_{b}\right| N_{b}\right)$. For $g_{b f}<0$, Eqs. (17) and (18) must be solved numerically. In the numerical calculations the LiebLiniger function $G(x)$ is modeled by an efficient Padé approximant based on the exact numerical determination of $G(x)[44]$.

As the effective potential of the problem is $E$ of Eq. (11), Eq. (16) together with the condition implicit in Eq. (19) minimizes the effective potential as a function of the two widths $\sigma_{b}$ and $\sigma_{f}$. A typical plot of the potential for $N_{b}$ $=100, N_{f}=10, g_{b}=0.01, g_{b f}=-0.2$, and $\lambda_{m}=1$ is shown in Fig. 1. Stable oscillations of the system are possible around the minimum. We shall study different features of these oscillations in the following.

As previously stressed, bosons are 1D under the condition $g_{b} n_{b} \ll 1$, which corresponds to $\sigma_{b} \gg g_{b} N_{b} / \sqrt{\pi}$. For a quite large width, namely, for $\sigma_{b} \gg N_{b} /\left(\sqrt{\pi} g_{b}\right)$, the bosons enter in the TG regime, where $g_{b} / n_{b} \gg 1$. The fermions are instead $1 \mathrm{D}$ under the condition $\left(\pi^{2} \lambda_{m} / 2\right) n_{f}^{2} \ll 1$, which corresponds to $\sigma_{f} \gg N_{f} \sqrt{\lambda_{m} \pi / 2}$.

We shall base the present study on the Gaussian variational approach described above, which, like any variational approach, should be reliable for the Bose-Fermi ground state studied in this paper. Moreover, the analytical variational solution provides interesting physical insight into the problem, as we shall see in the following. Also, the actual numerical solution of the full coupled dynamics is pretty complicated to implement for all cases reported in this paper in the various parameter ranges. Nevertheless, we find it worthwhile to compare the solution of the variational scheme with the ac-
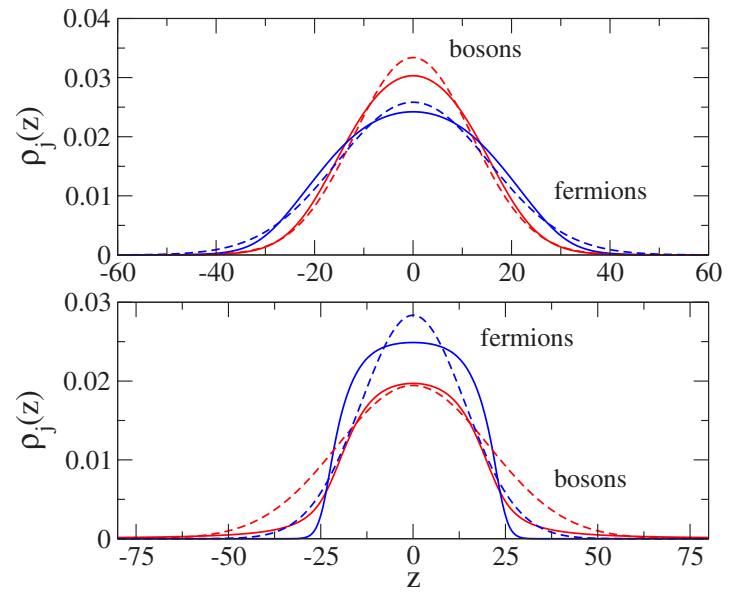

FIG. 2. (Color online) Probability density $\rho_{j}(z)=\left|\psi_{j}(z)\right|^{2} / N_{j}$ of bosons and fermions in the self-bound mixture with $g_{b}=0.01$ and $g_{b f}=-0.2$. In the upper panel, $N_{b}=100$ and $N_{f}=20$; in the lower panel, $N_{b}=300$ and $N_{f}=10$. Solid lines: numerical results. Dashed lines: variational results. Units as in Fig. 1.

curate numerical solution of Eqs. (5) and (6) in certain cases. We solved these numerically, using a imaginary-time integration method based on the finite-difference Crank-Nicholson scheme, as described in Ref. [45]. We discretize the meanfield equations using a time step $\Delta t=0.05$ and a space step $\Delta z=0.05$, and $z \in[-L / 2, L / 2]$ with $L=2000$. The boundary conditions are $\psi_{j}(-L / 2)=\psi_{j}(L / 2)=0$, with $j=b, f$. We start with broad Gaussians as initial wave functions. In the course of the imaginary-time evolution the self-bound mixture is quickly formed but, due to strong Pauli repulsion among identical spin-polarized fermions, the fermionic density profile extends to many hundredths of length's units. It is then essential to take a very large space interval $[-L / 2, L / 2]$ of integration to see that these long tails of the fermionic cloud are indeed decaying to zero.

In Fig. 2 we plot two sets of numerical results for the probability density in the quasi-BEC $1 \mathrm{D}$ regime. The figure shows that, for fixed values of the interaction strengths $g_{b}$ and $g_{b f}$, the axial width of the bosonic probability density becomes larger than the fermionic one by reducing the number $N_{f}$ of fermions and increasing the number $N_{b}$ of bosons. The figure shows that the variational approach can be used to give a reasonable estimation of the axial widths of the two clouds.

We now turn to a study of the Bose-Fermi system by using the variational approach, which enables us to explore quite easily all regimes and extract physically interesting analytical results. With the intention of illustrating the TG regime and the quasi-BEC Gross-Pitaevskii regime for bosons and 1D regime for fermions for a specific set of Bose-Fermi parameters. In Fig. 3 we plot $\sigma_{b}$ (solid line) and $\sigma_{f}$ (dashed line) from Eqs. (17) and (18) as a function of $\left|g_{b f}\right|$, with $g_{b f}<0$ for the values $N_{b}=100, N_{f}=20, g_{b}=0.01$, and $\lambda_{m}=1$ of the parameters. In the upper panel the linearlinear scale is employed while in the lower panel we report the same results on a log-log scale, to better visualize the TG regime and the quasi-BEC regimes. When their width takes values between $\sigma_{b}=g_{b} N_{b} / \sqrt{\pi} \simeq 0.56$ (dot-dashed line) and 

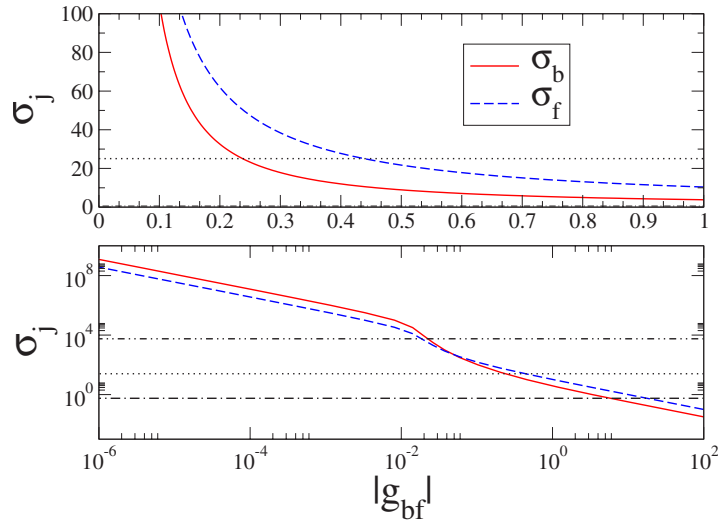

FIG. 3. (Color online) 1D self-bound Bose-Fermi droplet with repulsive bosons $\left(g_{b}>0\right)$. Axial widths $\sigma_{b}$ and $\sigma_{f}$ of the bosonic and fermionic widths as a function of the Bose-Fermi interaction strength $\left|g_{b f}\right|$, with $g_{b f}<0$. Mixture parameters: $N_{B}=100, N_{F}=20$, $\lambda_{m}=1$, and $g_{b}=0.01$. Upper panel: linear-linear scale. Lower panel: $\log$-log scale. Between the dot-dashed line and the dot-dot-dashed line the bosons are in the quasi-BEC 1D regime, while above the dot-dot-dashed line they are in the TG regime. Fermions are in the 1D regime above the dotted line. Units are as in Fig. 1.

$\sigma_{b}=N_{b} /\left(g_{b} \sqrt{\pi}\right) \simeq 5600$ (dot-dot-dashed line) bosons are in the quasi-BEC $1 \mathrm{D}$ regime, while they are in the TG $1 \mathrm{D}$ regime if $\sigma_{b}>5600$ (above the dot-dot-dashed line). Fermions are in the $1 \mathrm{D}$ regime when $\sigma_{f}>N_{f} \sqrt{\lambda_{m} \pi / 2} \simeq 25$, i.e., above the dotted line. For large $\left|g_{b f}\right|$, both the widths tend to zero corresponding to narrow soliton(s).

\section{QUASI-BOSE-EINSTEIN CONDENSATE ONE- DIMENSIONAL REGIME AND TONKS-GIRARDEAU REGIME}

The expression for the effective Lagrangian of the BoseFermi system given by Eqs. (11)-(14) is greatly simplified in the quasi-BEC $1 \mathrm{D}$ regime and also in the TG regime. In the quasi-BEC 1D regime one has $G(x) \simeq x$ and the expression for the effective energy $E$ of Eq. (11) can be evaluated analytically and written as

$$
E=\frac{1}{2} \frac{\alpha}{\sigma_{b}^{2}}+\frac{\beta}{\sigma_{b}}+\frac{1}{2} \frac{\gamma}{\sigma_{f}^{2}}-\frac{\delta}{\sqrt{\sigma_{b}^{2}+\sigma_{f}^{2}}},
$$

where $\alpha=N_{b}, \quad \beta=g_{b} N_{b}^{2} /(2 \sqrt{2 \pi}), \quad \gamma=\lambda_{m} N_{f}\left[1+2 \pi N_{f}^{2} /(3 \sqrt{3})\right]$, and $\delta=\left|g_{b f}\right| N_{b} N_{f} / \sqrt{\pi}$ with $g_{b f}=-\left|g_{b f}\right|$. Then at equilibrium one finds from Eq. (16) that $\sigma_{f}$ and $\delta$ can be written as functions of $\sigma_{b}$,

$$
\sigma_{f}=\gamma^{1 / 4} \frac{\sigma_{b}}{\left(\alpha+\beta \sigma_{b}\right)^{1 / 4}}
$$

and also

$$
\delta=\left(\frac{\alpha}{\sigma_{b}}+\beta\right)\left(1+\sqrt{\frac{\gamma}{\alpha+\beta \sigma_{b}}}\right)^{3 / 2} .
$$

Equation (22) implies that for any finite $\sigma_{b}$ and $\sigma_{f}$ one has $\delta>\beta$, i.e., $\left|g_{b f}\right|>\left|g_{b f}\right|_{\min }=\sqrt{2} g_{b} N_{b} /\left(4 N_{f}\right)$. Thus, for $g_{b}>0$ the
Bose-Fermi bright soliton exists only for $\left|g_{b f}\right|>\left|g_{b f}\right|$ min, while the system will not be bound $\left(\sigma_{b} \rightarrow \infty, \sigma_{f} \rightarrow \infty\right)$ for $\left|g_{b f}\right|<\left|g_{b f}\right|_{\text {min }}$. This last situation is, however, unphysical because for a very large $\sigma_{b}$ the system enters in the TG regime and Eq. (20) is no more valid.

When the Bose-Bose scattering length is zero $\left(g_{b}=0\right)$ then $\left|g_{b f}\right|_{\min }=0$. For $g_{b}<0$ (attractive Bose-Bose interaction), $\sigma_{b}$ is finite even for $\left|g_{b f}\right|_{\min }=0$, but it cannot exceed the value $\sigma_{b}=2 \sqrt{2 \pi} /\left(\left|g_{b}\right| N_{b}\right)$ with the corresponding value of $\sigma_{f}$ infinitely large. When $\left|g_{b f}\right|=0$ the bosonic cloud is localized $\left[\sigma_{b}=2 \sqrt{2 \pi} /\left(\left|g_{b}\right| N_{b}\right)\right]$ while the fermionic cloud is fully delocalized $\left(\sigma_{f}=+\infty\right)$ in the axial direction.

Coming back to the case of a repulsive Bose-Bose scattering length $\left(g_{b}>0\right)$, we observe that, after fixing $g_{b}$ and $N_{b}$, by reducing $\left|g_{b f}\right|$ the bosonic width $\sigma_{b}$ increases and the system enters in the TG regime, where $G(x) \simeq \pi^{2} / 3$ and the effective energy $E$ of Eq. (11) can also be evaluated analytically as

$$
E=\frac{1}{2} \frac{\alpha}{\sigma_{b}^{2}}+\frac{1}{2} \frac{\tilde{\beta}}{\sigma_{b}^{2}}+\frac{1}{2} \frac{\gamma}{\sigma_{f}^{2}}-\frac{\delta}{\sqrt{\sigma_{b}^{2}+\sigma_{f}^{2}}} .
$$

The quantities $\alpha, \gamma$, and $\delta$ are the same as in Eq. (20) while $\widetilde{\beta}=2 N_{b}^{3} \pi /(3 \sqrt{3})$. In this case the widths $\sigma_{b}$ and $\sigma_{f}$ at the equilibrium can also be determined analytically. They are

$$
\begin{gathered}
\sigma_{b}=\frac{\alpha+\tilde{\beta}}{\delta}\left(1+\sqrt{\frac{\gamma}{(\alpha+\tilde{\beta})}}\right)^{3 / 2}, \\
\sigma_{f}=\gamma^{1 / 4} \frac{(\alpha+\tilde{\beta})^{3 / 4}}{\delta}\left(1+\sqrt{\frac{\gamma}{(\alpha+\tilde{\beta})}}\right)^{3 / 2},
\end{gathered}
$$

and both increase as $\delta$ decreases, and only when $\delta=\left|g_{b f}\right|$ $=0$ the two widths become infinitely large. Thus we conclude that for any finite value of $\left|g_{b f}\right|$ and $g_{b}>0$ there exists a Bose-Fermi bright soliton, the existence of this bright soliton being guaranteed by the behavior of the bosonic energy term in the TG regime.

In Fig. 4 we show the axial widths $\sigma_{b}$ and $\sigma_{f}$ of the Bose-Fermi mixture with an attractive Bose-Bose scattering length $\left(g_{b}<0\right)$. We choose $N_{b}=50, N_{f}=10$, and $g_{b}=-0.01$ and plot the widths as a function of $\left|g_{b f}\right|$. The figure shows that, as $\left|g_{b f}\right|$ goes to zero, the Fermi width $\sigma_{f}$ is much larger than the Bose width $\sigma_{b}$. In fact, as previously shown, at $\left|g_{b f}\right|=0$, one finds $\sigma_{f}=+\infty$ while $\sigma_{b}=2 \sqrt{2 \pi} /\left(\left|g_{b}\right| N_{b} \mid\right)$. For large values of $\left|g_{b f}\right|$, the Fermi width $\sigma_{f}$ quickly decreases and reaches the value $\sigma_{f}=N_{f} \sqrt{\lambda_{m} \pi / 2} \approx 12.5$ (dotted line) below which the Fermi system is no more strictly one dimensional.

\section{SINGLE FERMIONIC ATOM IN THE BOSE CLOUD}

The existence of the Bose-Fermi bright soliton for both attractive and repulsive Bose-Bose interaction, provided there is an attractive Bose-Fermi interaction, even vanishingly small, is due to the $1 \mathrm{D}$ effect of quantum fluctuations described by the Lieb-Liniger function $G(x)$. To understand 


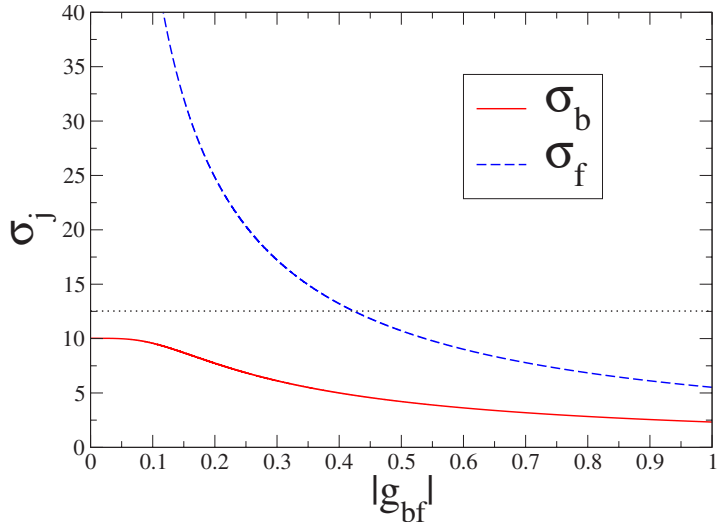

FIG. 4. (Color online) 1D self-bound Bose-Fermi droplet with attractive bosons $\left(g_{b}<0\right)$. Axial widths $\sigma_{b}$ and $\sigma_{f}$ of the bosonic and fermionic widths as a function of the Bose-Fermi interaction strength $\left|g_{b f}\right|$, with $g_{b f}<0$. Mixture parameters: $N_{b}=50, N_{f}=10$, $\lambda_{m}=1$, and $g_{b}=-0.01$. Fermions are in the $1 \mathrm{D}$ regime above the dotted line. Units are as in Fig. 1.

this result further we consider the case of a single fermionic atom $\left(N_{f}=1\right)$ interacting with the Bose cloud. In this case the exact stationary Schrödinger equation of the single-particle wave function $\psi_{f}(z)$ of the fermion is given by

$$
\left(-\lambda_{m} \partial_{z}^{2}-\left|g_{b f}\right|\left|\psi_{b}\right|^{2}\right) \psi_{f}=\epsilon \psi_{f}
$$

where $\epsilon$ is the energy of the fermionic bound state under the effective potential well $V_{\text {eff }}(z)=-\left|g_{b f}\right|\left|\psi_{b}(z)\right|^{2}$ determined by the Bose cloud. We know that for $g_{b}<0$ the pure 1D Bose system supports a bright soliton described by $\psi_{b}(z)$ $=\frac{\left(\left|g_{b}\right| N_{b}\right)^{1 / 2}}{2} \operatorname{sech}\left(\frac{\left|g_{b}\right| z}{2}\right)$. Guided by this result, in the present case of repulsive bosons $\left(g_{b}>0\right)$ attracted by a single fermion $g_{b f}<0$ we adopt for the bosonic field $\psi_{b}(z)$ the following ansatz:

$$
\psi_{b}(z)=\frac{N_{b}^{1 / 2}}{(2 \xi)^{1 / 2}} \operatorname{sech}\left(\frac{z}{\xi}\right)
$$

with $\xi$ the variational width of the bosonic cloud. This ansatz is also suggested by the existence of the analytical solution for the eigenvalue of the corresponding Eq. (26) for the fermionic bound state. One finds [46]

$$
\epsilon(\xi)=-\frac{1}{2 \xi^{2}}\left(1+\left|g_{b f}\right| N_{b} \xi-\sqrt{1+2\left|g_{b f}\right| N_{b} \xi}\right)^{2}
$$

The energy $\epsilon(\xi)$ is the sum of the kinetic energy of the fermionic atom and of the interaction energy between the fermionic atom and the bosonic cloud.

The total energy of the system is then the effective energy of the Bose cloud calculated from the Lagrangian density (2) added to the energy $\epsilon(\xi)$ of the Fermi atom and is given by

$$
E=\frac{N_{b}}{3 \xi^{2}}+\frac{N_{b}^{3}}{8 \xi^{2}} \int_{-\infty}^{+\infty} \operatorname{sech}^{6}(y) G\left(\frac{g_{b} \xi}{N_{b} \operatorname{sech}^{2}(y)}\right) d y+\epsilon(\xi) .
$$

The existence of the Bose-Fermi bright soliton depends on the existence of a local minimum of this total energy $E$ $=E(\xi)$ of the system. From Eq. (29) we find that $E(\xi)$ $\sim-\frac{\left|g_{b}\right| N_{b}}{2 \xi}$ as $\xi \rightarrow+\infty$, since for large $\xi$ 's $G\left(\frac{g_{b} \xi}{N_{b} \operatorname{sech}^{2}(y)}\right) \simeq \frac{\pi^{2}}{3}$ for any value of $y$. Thus, for $\xi \rightarrow+\infty$, the energy goes to zero through negative values. Again, from Eq. (29) we see that $E(\xi) \rightarrow+\infty$ as $\xi \rightarrow 0$ and therefore we conclude that the function $E(\xi)$, being positive at the origin and vanishingly small but negative, at large $\xi$ 's, must possess a negative local minimum that is also the global minimum of the energy. This implies that there is always a finite value of $\xi$ that minimizes the total energy. As previously stressed, this behavior strictly depends on the properties of the Lieb-Liniger function $G(x)$ for large $x$ and, as a consequence, the Bose-Fermi bright soliton exists for any negative value $g_{b f}$, even for $N_{f}=1$.

\section{COLLECTIVE OSCILLATIONS OF THE BOSE-FERMI BRIGHT SOLITON}

After having established the existence of stationary bright solitons in a degenerate Bose-Fermi mixture, we study two types of small oscillations of this system around the stable equilibrium position and calculate their frequencies. The first is the stable breathing oscillation of the system around its mean position and the second describes the stable small oscillations once the centers of the Fermi and Bose clouds are slightly displaced with respect to each other. Given the effective Lagrangian (9), the problem of small oscillations is solved by expanding the kinetic energy (10) and the potential energy (11) around the equilibrium solution up to quadratic terms. In this way we extract [43] the frequencies $\omega$ of the collective breathing modes of the Bose and Fermi clouds from the eigenvalue equation,

$$
\left(\begin{array}{cc}
\frac{\partial^{2} E}{\partial \sigma_{b}^{2}} & \frac{\partial^{2} E}{\partial \sigma_{b} \sigma_{f}} \\
\frac{\partial^{2} E}{\partial \sigma_{b} \sigma_{f}} & \frac{\partial^{2} E}{\partial \sigma_{f}^{2}}
\end{array}\right)-\omega^{2}\left(\begin{array}{cc}
N_{b} & 0 \\
0 & N_{f} \lambda_{m}
\end{array}\right)=0,
$$

where the partial derivatives must be calculated at the equilibrium $\left(\sigma_{b}, \sigma_{b}\right)$, where $\sigma_{b}$ and $\sigma_{f}$ are the Bose and Fermi widths obtained from Eqs. (17) and (18). The two frequencies $\omega_{1}$ and $\omega_{2}$ then read

$$
\omega_{1,2}=\sqrt{\frac{N_{b} \frac{\partial^{2} E}{\partial \sigma_{f}^{2}}+\lambda_{f} N_{f} \frac{\partial^{2} E}{\partial \sigma_{b}^{2}} \pm \sqrt{\Delta}}{2 \lambda_{m} N_{b} N_{f}}},
$$

where

$$
\Delta=\left(N_{b} \frac{\partial^{2} E}{\partial \sigma_{f}^{2}}+\lambda_{m} N_{f} \frac{\partial^{2} E}{\partial \sigma_{b}^{2}}\right)^{2}-4 \lambda_{m} N_{b} N_{f} K_{G},
$$

and $K_{G}$ is the Gaussian curvature of Eq. (19). 


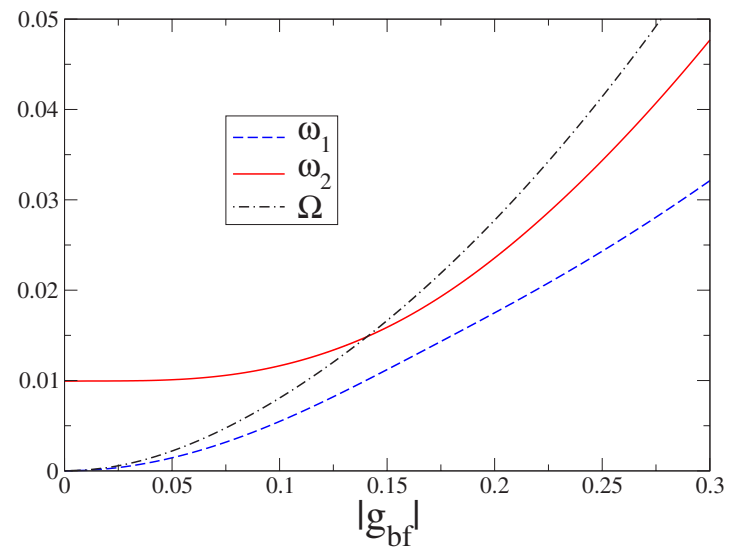

FIG. 5. (Color online) Collective breathing frequencies $\omega_{1}$ and $\omega_{2}$ of the self-bound Bose-Fermi droplet with attractive bosons $\left(g_{b}<0\right) . \Omega$ is the harmonic frequency of the displacement $\left|z_{1}-z_{2}\right|$ of the centers of mass of the Bose and Fermi clouds. Parameters are as in Fig. 4. Frequencies are in units of the frequency $\omega_{\perp}$ of transverse harmonic confinement.

In addition, by taking into account the "reduced mass" $\lambda_{m} N_{b} N_{f} /\left(N_{b}+\lambda_{m} N_{f}\right)$, one can derive the frequency $\Omega$ of harmonic oscillation of the relative distance $\left|z_{b}-z_{f}\right|$ (displacement) of the two clouds from the equation

$$
\frac{\lambda_{m} N_{b} N_{f}}{N_{b}+\lambda_{m} N_{f}} \Omega^{2}=\frac{\partial^{2} E}{\partial\left(\mid z_{b}-z_{f}\right)^{2}} .
$$

The result is

$$
\Omega=\sqrt{\frac{2\left|g_{b f}\right|\left(N_{b}+\lambda_{m} N_{f}\right)}{\pi\left(\sigma_{b}^{2}+\sigma_{f}^{2}\right)^{3 / 4}}},
$$

where again $\sigma_{b}$ and $\sigma_{f}$ are the Bose and Fermi widths at equilibrium, where $z_{b}=z_{f}$.

In Fig. 5 we plot the frequencies $\omega_{1}$ and $\omega_{2}$ of the coupled breathing modes of the Bose and Fermi clouds by choosing the same parameters of Fig. 4, namely, $N_{b}=50, N_{f}=10, \lambda_{m}$ $=1$, and $g_{b}=-0.01$. The frequencies are plotted as a function of the Bose-Fermi strength $\left|g_{b f}\right|$. For $\left|g_{b f}\right|=0$, the frequencies are decoupled: $\omega_{1}$ is the frequency of the fermionic axial breathing mode and $\omega_{2}$ is the frequency of the bosonic axial breathing mode. Without a Bose-Fermi interaction the Fermi cloud is delocalized $\left(\sigma_{f}=\infty\right)$ and its breathing frequency is $\omega_{1}=0$, while the Bose cloud remains localized (due to the negative Bose-Bose strength $g_{b}$ ) and its breathing frequency $\omega_{2}$ remains finite and is equal to $\omega_{2}=g_{b}^{4} N_{b}^{4} /\left(16 \pi^{2}\right)$. Figure 5 shows that both the breathing frequency $\omega_{1}$ and the harmonic frequency $\Omega$ of the displacement $\left|z_{1}-z_{2}\right|$ start from zero and grow as $\left|g_{b f}\right|$ increases.

\section{CONCLUSION}

We have studied a degenerate 1D Bose-Fermi mixture by using the quantum hydrodynamics. We find that for attractive Bose-Fermi interaction $\left(g_{b f}<0\right)$ the ground state of the system is a self-bound Bose-Fermi droplet. The nonexistence of a threshold in the strength of an attractive Bose-Fermi interaction for the formation of a Bose-Fermi bright soliton in one dimension is confirmed in the case of a single Fermi atom immersed in a degenerate Bose gas with repulsive Bose-Bose interaction. We also calculate the frequencies of stable oscillation of the Bose-Fermi bright soliton. Such a Bose-Fermi bright soliton is similar to a recently studied Bose-Bose bright soliton bound through an attractive interspecies interaction [47].

In view of the recent experimental studies of a degenerate $1 \mathrm{D}{ }^{87} \mathrm{Rb}$ gas $[29,30]$ and the successful identification of the quasi-BEC and TG regime in it and the observation of the degenerate Bose-Fermi mixture in ${ }^{6} \mathrm{Li}-{ }^{7} \mathrm{Li}[16,17],{ }^{40} \mathrm{~K}-{ }^{87} \mathrm{Rb}$ [13], ${ }^{6} \mathrm{Li}^{23} \mathrm{Na}$ [18], etc. by different groups, the experimental realization of a Bose-Fermi bright soliton seems possible with present technology. The most attractive procedure seems to make use of an experimentally observed Feshbach resonance [48] in a Bose-Fermi mixture. The 1D Bose-Fermi mixture must be created in an axial harmonic trap and the Bose-Fermi interaction must be turned from repulsive to attractive by manipulating a background magnetic field. At the same time the axial harmonic trap on the system should be removed. Upon removal of the axial trap, the result is the formation of a single or a train of bright solitons as in the experiment with the degenerate Bose system of ${ }^{7} \mathrm{Li}$ atoms [17] or as in a numerical simulation in a degenerate BoseFermi mixture [19]. By choosing numbers of atoms and interatomic strengths as suggested in the present paper, one obtains a single Bose-Fermi bright soliton and can study its static and dynamical properties.

\section{ACKNOWLEDGMENTS}

This work is partially supported by the FAPESP and CNPq of Brazil.
[1] L. P. Pitaevskii and S. Stringari, Bose-Einstein Condensation (Oxford University Press, Oxford, 2003).

[2] K. M. O'Hara, S. L. Hemmer, M. E. Gehm, S. R. Granade, and J. E. Thomas, Science 298, 2179 (2002).

[3] M. Greiner, C. A. Regal, and D. S. Jin, Nature (London) 426, 537 (2003).

[4] S. Jochim, M. Bartenstein, A. Altmeyer, G. Hendl, S. Riedl, C. Chin, J. H. Denschlag, and R. Grimm, Science 302, 2101
(2003).

[5] T. Bourdel et al., Phys. Rev. Lett. 93, 050401 (2004).

[6] K. Mølmer, Phys. Rev. Lett. 80, 1804 (1998).

[7] N. Nygaard and K. Mølmer, Phys. Rev. A 59, 2974 (1999).

[8] L. Viverit, C. J. Pethick, and H. Smith, Phys. Rev. A 61, 053605 (2000).

[9] L. Viverit, Phys. Rev. A 66, 023605 (2002).

[10] K. K. Das, Phys. Rev. Lett. 90, 170403 (2003). 
[11] X. J. Liu, M. Modugno, and H. Hu, Phys. Rev. A 68, 053605 (2003); S. T. Chui and V. N. Ryzhov, ibid. 69, 043607 (2004); S. K. Adhikari, ibid. 70, 043617 (2004).

[12] D. W. Wang, Phys. Rev. Lett. 96, 140404 (2006).

[13] G. Roati, F. Riboli, G. Modugno, and M. Inguscio, Phys. Rev. Lett. 89, 150403 (2002).

[14] M. Modugno, F. Ferlaino, F. Riboli, G. Roati, G. Modugno, and M. Inguscio, Phys. Rev. A 68, 043626 (2003).

[15] C. Ospelkaus, S. Ospelkaus, K. Sengstock, and K. Bongs, Phys. Rev. Lett. 96, 020401 (2006).

[16] F. Schreck, L. Khaykovich, K. L. Corwin, G. Ferrari, T. Bourdel, J. Cubizolles, and C. Salomon, Phys. Rev. Lett. 87, 080403 (2001).

[17] A. G. Truscott, K. E. Strecker, W. I. McAlexander, G. B. Partridge, and R. G. Hulet, Science 291, 2570 (2001).

[18] Z. Hadzibabic, C. A. Stan, K. Dieckmann, S. Gupta, M. W. Zwierlein, A. Gorlitz, and W. Ketterle, Phys. Rev. Lett. 88, 160401 (2002).

[19] T. Karpiuk et al., Phys. Rev. Lett. 93, 100401 (2004); T. Karpiuk, M. Brewczyk, and K. Rzazewski, Phys. Rev. A 73, 053602 (2006).

[20] S. K. Adhikari, Phys. Rev. A 72, 053608 (2005).

[21] J. Santhanam, V. M. Kenkre, and V. V. Konotop, Phys. Rev. A 73, 013612 (2006).

[22] L. Salasnich, J. Math. Phys. 41, 8016 (2000).

[23] D. S. Petrov, M. Holzmann, and G. V. Shlyapnikov, Phys. Rev. Lett. 84, 2551 (2000).

[24] E. H. Lieb and W. Liniger, Phys. Rev. 130, 1605 (1963).

[25] M. Girardeau, J. Math. Phys. 1, 516 (1960); M. Girardeau, Phys. Rev. 139, B500 (1965); L. Tonks, Phys. Rev. 50, 955 (1936).

[26] J. B. McGuire, J. Math. Phys. 5, 622 (1964).

[27] F. Calogero and A. Degasperis, Phys. Rev. A 11, 265 (1975).

[28] L. Salasnich, J. Phys. B 39, 1743 (2006).

[29] T. Kinoshita, T. Wenger, and D. S. Weiss, Science 305, 1125 (2004); B. Paredes, A. Widera, V. Murg, O. Mandel, S. Folling, I. Cirac, G. V. Shlyapnikov, T. W. Hansch, and I. Bloch, Nature (London) 429, 277 (2004).

[30] T. Kinoshita, T. Wenger, and D. S. Weiss, Nature (London) 440, 900 (2006).

[31] B. DeMarco and D. S. Jin, Science 285, 1703 (1999); K. E. Strecker, G. B. Partridge, and R. G. Hulet, Phys. Rev. Lett. 91, 080406 (2003); K. M. O'Hara, S. L. Hemmer, M. E. Gehm, S. R. Granade, and J. E. Thomas, Science 298, 2179 (2002).
[32] S. K. Adhikari, J. Phys. B 38, 3607 (2005).

[33] E. B. Kolomeisky, T. J. Newman, J. P. Straley, and X. Qi, Phys. Rev. Lett. 85, 1146 (2000).

[34] A. Minguzzi, P. Vignolo, M. L. Chiofalo, and M. P. Tosi, Phys. Rev. A 64, 033605 (2001).

[35] L. Salasnich, A. Parola, and L. Reatto, Phys. Rev. A 70, 013606 (2004); L. Salasnich, A. Parola, and L. Reatto, ibid. 72, 025602 (2005).

[36] L. Salasnich, A. Parola and L. Reatto, Phys. Rev. A 69, 045601 (2004).

[37] L. Salasnich, Laser Phys. 12, 198 (2002); L. Salasnich, A. Parola, and L. Reatto, Phys. Rev. A 65, 043614 (2002).

[38] K. K. Das, Phys. Rev. A 66, 053612 (2002).

[39] M. Olshanii, Phys. Rev. Lett. 81, 938 (1998).

[40] E. H. Lieb, R. Seiringer, and J. Yngvason, Phys. Rev. Lett. 91, 150401 (2003); E. H. Lieb, R. Seiringer, and J. Yngvason, Commun. Math. Phys. 244, 347 (2004).

[41] M. D. Girardeau and E. M. Wright, Phys. Rev. Lett. 84, 5239 (2000).

[42] V. M. Perez-Garcia, H. Michinel, J. I. Cirac, M. Lewenstein, and P. Zoller, Phys. Rev. A 56, 1424 (1997); D. Anderson, ibid. 27, 3135 (1983); H. T. C. Stoof, J. Stat. Phys. 87, 1353 (1997).

[43] L. Salasnich, Int. J. Mod. Phys. B 14, 1 (2000).

[44] The Lieb-Liniger function $G(x)$ is such that for $x \ll 1$ one finds $G(x) \simeq x+B x^{3 / 2}+\eta x^{2}$, where $B=4 /(3 \pi)$ and $\eta=0.0648$ (from numerics). Instead for $x \gg 1$ one has $G(x) \simeq\left(\pi^{2} / 2\right) x^{2} /(x+2)^{2}$. The Padé approximant we use is $G(x)=\left(x+A x^{3}\right) /\left[1+B x^{1 / 2}\right.$ $\left.+C x(x+2)^{2}\right]$, where $A=\pi^{2}\left(B^{2}-\eta\right) / 12$ and $C=\left(B^{2}-\eta\right) / 4$. For further details see Ref. [35].

[45] E. Cerboneschi, R. Mannella, E. Arimondo, and L. Salasnich, Phys. Lett. A 249, 495 (1998); L. Salasnich, A. Parola, and L. Reatto, Phys. Rev. A 64, 023601 (2001); L. Salasnich and B. A. Malomed, ibid. 74, 053610 (2006).

[46] L. D. Landau and E. M. Lifshitz, Course of Theoretical Physics, in Quantum Mechanics: Non-Relativistic Theory (Pergamon Press, Oxford, 1977), Vol. 3.

[47] S. K. Adhikari, Phys. Lett. A 346, 179 (2005); V. M. PerezGarcia and J. B. Beitia, Phys. Rev. A 72, 033620 (2005).

[48] C. A. Stan, M. W. Zwierlein, C. H. Schunck, S. M. F. Raupach, and W. Ketterle, Phys. Rev. Lett. 93, 143001 (2004); S. Inouye, J. Goldwin, M. L. Olsen, C. Ticknor, J. L. Bohn, and D. S. Jin, ibid. 93, 183201 (2004). 